\title{
Alginate Hydrogel for 3D Bioprinting
}

Ying $\mathrm{Mei}^{1,2}$

${ }^{1 .}$ Department of Bioengineering, Clemson University, Clemson, SC, USA

2. Department of Regenerative Medicine and Cell Biology, Medical University of South Carolina, Charleston, SC, USA

3D bioprinting holds remarkable potential for the rapid fabrication of tissue engineering constructs with high fidelity. As a biocompatible biomaterial that can robustly form hydrogels in the physiological condition, alginate has been widely utilized in bioprinting applications [1]. To this end, my lab has explored the application of alginate in both indirect and direct 3D biofabrication. In the case of indirect fabrication, hydrogel molds with defined structures were 3D printed and used to facilitate the formation of scaffold-free tissues with predetermined shapes and patterns (e.g. toroids, honeycombs) [2]. For this application, we utilized native alginate hydrogels due to their non-biodegradable, bio-inert, and biocompatible properties. For direct fabrication, we utilized chemically modified alginates to create conducive environments for cell adhesion, proliferation and tissue formation.

For indirect biofabrication, we have demonstrated the use of a customized 3D printer to prepare biocompatible, bio-inert hydrogel molds for the production of scaffold-free tissue engineering constructs [2]. As shown in Figure 1, alginate microdroplets were printed upon a calcium containing substrate to form ring-shaped molds with high-resolution. Subsequently, human tissue spheroids composed of 1:1 endothelial cells and smooth muscle cells were robotically seeded upon the alginate molds, where they rapidly fused to form toroid-shaped tissue constructs. This approach serves as a proof-of-concept in using unmodified alginate hydrogels for the production of open-structured 3D molds. However, further modifications will be required to produce nonopen-structured molds that would allow for more complicated shapes.

In contrast, direct methods allow for the direct fabrication of complex structures using a cellinteractive bioink [3]. While native alginate is a bioinert material (i.e. lacks cell-adhesive moieties) with limited biodegradability, recent advances in biomaterials have allowed us to prepare cell-adhesive alginate hydrogels with predictable rates of biodegradation. We have systematically investigated the effects of several material properties of alginate hydrogels (i.e. viscosity and density) in order to identify a suitable range of printable solutions. Through the process of optimization, four alginate solutions with different levels of biodegradability and containing human adipose-derived stem cells (hADSCs) were printed into cell-laden latticestructures (Figure 2), creating a foundation for the further development of tunable alginate-based bioink.

While bioprinting has emerged as a powerful tool, the current lack of suitable bioinks has limited its applications in a clinical setting. The results shown above represent our initial efforts to use/engineer alginate for bioprinting applications. Our current research focuses on the development of alginate-based bioink that can promote neovasculariuzation after transplantation. The ability to 3D bioprint vascularized tissue engineering constructs would greatly accelerate the applications of 3D bioprinting technology in disease modeling and regenerative medicine [4]. 


\section{References:}

[1] D Richards, Y Tan, J Jia, et al, Isr J Chem 53 (2013), p. 805-814.

[2] Y Tan, D Richards, T Trusk, et al, Biofabrication 6 (2014), p. 024111.

[3] J Jia, D Richards, S Pollard, et al, Acta Biomater 10(10): 4323-4331

[4] The work is supported by the National Science Foundation (NSF - EPS-0903795), the startup funds from Clemson University, and the National Institutes of Health (8P20 GM103444). This study used the services of the Morphology, Imaging and Instrumentation Core, which is supported by NIH-NIGMS P30 GM103342 to the South Carolina COBRE for Developmentally Based Cardiovascular Diseases.

Step 1: Microdrops of native alginate were printed out to form a ring pattern.

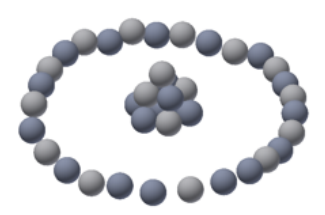

Step 2: Print layer by layer to form a 3D structure.

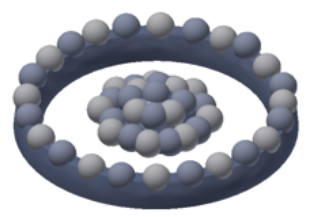

Step 3: Seeding spheroids within the ring structure

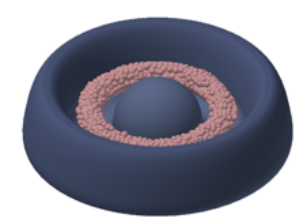

Step 4: Spheroids fuse together, forming a vascular tissue unit

Figure 1. Schematic representation of 3D alginate hydrogel printing for tissue unit fabrication using vascular spheroids (i.e., containing smooth muscle cells and endothelial cells).

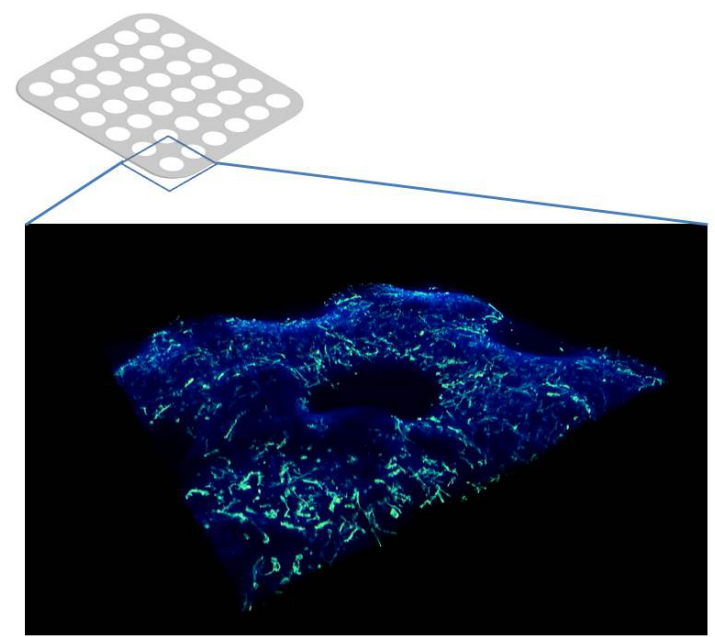

Figure 2. A computer-rendered 3-D picture of a portion of the printed lattice structure showing spreading hADSCs within the hydrogel. 